\title{
Subnetwork partitioning and section restoration in translucent optical networks
}

Ezhan Karasan, Mustafa Arisoylu 


\title{
Subnetwork Partitioning and Section Restoration in Translucent Optical Networks
}

\author{
Ezhan $\operatorname{Karasan}^{a}$ and Mustafa Arisoylu ${ }^{b}$ \\ ${ }^{a}$ Department of Electrical and Electronics Engineering \\ Bilkent University \\ Bilkent 06800, Ankara, Turkey \\ ${ }^{b}$ Department of Electrical and Computer Engineering \\ University of California at San Diego \\ La Jolla, CA 92093-0407, USA
}

\begin{abstract}
We discuss the problem of designing translucent optical networks composed of restorable, transparent subnetworks interconnected via transponders. We formulate the problem of designing restorable subnetworks in translucent networks as an Integer Linear Programming (ILP) problem, where the subnetworks are determined subject to the constraints that each subnetwork satisfies size limitations and it is 2-connected. A greedy heuristic algorithm for the same problem is also proposed for planar network topologies.

We propose section restoration for translucent networks where failed connections are rerouted inside the subnetwork which contains the failed link. The network design problem of determining working and restoration capacities with section restoration is formulated as an ILP problem. Numerical results show that section restoration generates fiber costs which are close to those with the path restoration technique for the mesh topologies used in this study. It is also shown that the number of transponders with the translucent optical network is substantially reduced compared to opaque networks.
\end{abstract}

Keywords: Translucent optical networks, subnetwork partitioning, optical restoration

\section{INTRODUCTION}

Multiwavelength optical networks are currently deployed widely in long-distance core networks. There are several architectures suitable for optical networks each involving complex combinations of optical and electronic devices. All these architectures have the optical fiber as the transmission medium and contain some form of optical cross-connects (OXC) interconnecting these fibers.

In transparent or all-optical networks architecture, a connection goes through the network over a completely optical path. ${ }^{1,2}$ This path consists of point-to-point optical links interconnected by all-optical nodes. Wavelength selective cross-connects (WSXC) can switch an incoming connection on some wavelength onto the same wavelength in any of its outgoing fibers. In networks utilizing WSXCs, connections must satisfy the wavelength continuity constraint, i.e., a connection must remain on the same wavelength on all links along its path. On the other hand, wavelength interchanging cross-connects (WIXC) employ optical wavelength conversion in order to switch an incoming connection onto any wavelength in any outgoing fiber. As such, they enable more efficient packing of wavelengths onto the fibers by eliminating wavelength conflicts. Optical wavelength converter technologies have relatively high costs making WIXCS significantly more expensive compared to WSXCs. ${ }^{3}$ Performance of all-optical networks with and without wavelength converters have been studied extensively in the literature. ${ }^{4-6}$

Second type of optical networks is the opaque network architecture. ${ }^{7}$ In opaque networks, a connection passes through optical/electronic/optical converters, called transponders, at each node. Opaque networks may utilize optical or electronic form of switching at intermediate nodes. Optical networks that are deployed today are typically opaque, and they use electronic cross-connects (EXC). ${ }^{8}$

E.K.: e-mail: ezhan@ee.bilkent.edu.tr, M.A.: e-mail: marisoyl@ucsd.edu 
One of the main advantages of all-optical networks is transparency. There are various levels of transparency delivered by all-optical networks such as bit rate, protocol, modulation format and analog/digital transparencies. Transparency enables easier deployment of new technologies. However, transparency also brings a cost due to accumulated impairments in optical transmission and switching systems requiring regeneration of optical signals.

Because of the limitations of building national-scale multi-vendor all-optical networks and the high cost of opaque networks, a new optical network architecture is recently considered. In this formation, there are islands of transparency, i.e., all-optical subnetworks, each having a limited geographic size. ${ }^{8-10}$ These subnetworks are interconnected to each other via non-transparent transponders forming a translucent optical network. Translucent networks have several advantages over previously known architectures: easier multi-vendor interoperability, reduced transponder costs, simpler network management, scalable network architecture and faster restoration times.

In this paper, we discuss two interrelated problems in the design of translucent networks. First, we describe how a given topology can be partitioned into transparent subnetworks such that each subnetwork is restorable against all possible single-link failures. We formulate this problem as an ILP problem where the network is partitioned into smallest number of subnetworks subject to the constraints that each subnetwork satisfies some size constraints and it is 2-connected. A greedy heuristic algorithm for the same problem is also proposed for planar network topologies.

After the partitioning problem is solved, the network design problem of determining working and restoration capacities using the proposed section restoration in translucent networks is studied. Resulting optimization problem is formulated as an ILP problem. Although network partitioning and capacity design problems are coupled to each other, we consider them separately because of the large computational complexity of the joint problem. The network partitioning problem is solved by only using the topology of the network independent of the traffic, since partitioning must be done at the initial network deployment phase. On the other hand, solution of the capacity design problem depends on the offered traffic since network resources can be dynamically provisioned with response to changes in the traffic demand.

After presenting the proposed section restoration technique for translucent network architecture in Section 2, we discuss the network partitioning problem in Section 3. In Section 4, the network design problem of computing working and restoration capacities is considered. Conclusions of the paper are presented in Section 5.

\section{SECTION RESTORATION IN TRANSLUCENT OPTICAL NETWORKS}

As optical networking becomes more commonplace in core transport networks optical switching technologies will be introduced in more network nodes. When the number of optical nodes in the network is large, the design and management of the network become more complicated. Translucent networks partition a network topology into multiple subnetworks. Each of these subnetworks can be designed and managed separately, simplifying these problems substantially. This results in a more scalable network architecture: as the number of nodes in the network increases the number of subnetworks will increase, but not sizes of these subnetworks.

Translucent networks also provide simplified operation, administration and management (OAM). One of the most important OAM functionalities is the restoration. In translucent networks, the restoration function can be managed by multiple restoration managers (RM), one for each subnetwork. Each RM is held accountable for restoring failures within its own subnetwork. Each RM keeps a separate database containing all information for the connections routed through its own subnetwork. In this paper, we consider restoration in the optical layer in response to single-link failures. When a link fails, corresponding RM reroutes all failed connections within its subnetwork without communicating with other RMs. Therefore, no interworking is necessary between RMs for restoring connections after a failure. This restoration technique is called section restoration. Rerouting techniques similar to the section restoration have been proposed recently in the literature. ${ }^{11,12}$

In the section restoration, when a link within subnetwork $S_{i}$ fails, the transponders located at the boundaries of $S_{i}$ detect the failure of each affected connection without requiring any optical monitoring. If a failed connection has its source and/or destination nodes in $S_{i}$, then the failure is detected by the transceivers at the source/destination nodes. Failure information is forwarded to the RM for $S_{i}$ which handles the restoration of all 
failed connections. Each failed connection is rerouted between its entry and exit points of subnetwork $S_{i}$ such that the restoration path is link-disjoint from the working path within $S_{i}$.

Section restoration is a quasi-distributed technique where after a link failure a single RM reroutes the affected traffic independent of other RMs. Since the size of each subnetwork is relatively small, propagation of failure information and reconfiguration of OXCs can be accomplished in shorter time with section restoration compared to path restoration where failed connections are rerouted between the source and destination nodes. Restoration path may use a different wavelength than the working path within the failed subnetwork since transponders and tunable laser/receivers can perform wavelength conversion.

With section restoration, only the nodes at the boundaries of the subnetworks need to carry restoration functions. Interior nodes need to know nothing about the restoration function except that they need to report failures of adjacent links to RM.

In this paper, we consider preplanned restoration where working and restoration link capacities are computed for static traffic demand such that all connections can be restored in the case of any single-link failure. Path restoration, where failed connections are rerouted between the source and destination nodes, have been studied extensively in the literature. Routing and capacity planning for path restoration is discussed. ${ }^{13-16}$ Section restoration is a special form of path rerouting where paths are restricted to subnetworks.

In the next section, we discuss the problem of partitioning the network into subnetworks subject to size and connectivity constraints.

\section{NETWORK PARTITIONING}

Subnetworks in the translucent optical network may need to satisfy several properties. First, each subnetwork should be 2-connected, i.e., there should exist at least two link-disjoint paths between any two nodes in the same subnetwork. This is a crucial constraint for guaranteeing full restoration against any single-link failure. We assume that the network topology corresponds to a graph that can be partitioned into 2-connected subnetworks. A trivial necessary condition for this is that the network topology itself is 2-connected.

Next, the size of the subnetworks may need to be below some certain thresholds. If each subnetwork contains small number of links and nodes, RMs deal with smaller databases; and this leads to a more scalable architecture with shorter restoration times. In this paper, the lengths of working and restoration paths between any two nodes in the same subnetwork can be at most $R_{\max }$. We also restrict the number of nodes in a subnetwork to $N_{\max }$ and the number of links to $L_{\max }$ so that computational complexity of the network design problem for each subnetwork is bounded. With small-sized subnetworks, restoration capacities are used inefficiently since section restoration in this case resembles link rerouting. Therefore, the number of subnetworks is minimized, leading to subnetworks with sizes close to the constraints.

For restoration purposes each link should belong to only one subnetwork, i.e., when a link fails, RM of the related subnetwork will be responsible for restoration of all failed connections. However, for partitioning purposes, a link may be shared by two or more subnetworks in order to satisfy 2-connectivity for each subnetwork. This can be described by introducing a two-layer structure. In the restoration layer, each link belongs to a single subnetwork, i.e., when a link fails the corresponding RM handles the failure. On the other hand, in overlapped subnetworks layer some links may be shared between two or more subnetworks so that each subnetwork is 2connected. The links shared in the overlapped subnetworks layer may be carrying restored traffic resulting from a failure in one of the sharing subnetworks. Since we only consider single-link failures, a shared link can be used by rerouted traffic directed by at most one RM at any given time.

Based on the above constraints, two solutions for network partitioning problem are presented below, first based on an ILP formulation and the other based on a greedy heuristic algorithm.

\subsection{Partitioning with ILP Formulation}

The ILP formulation for the network partitioning problem consists of two parts. First, we compute all linkdisjoint paths between each node pair, and then the network is divided into subnetworks using the outputs 
of the first part. Link-disjoint paths are used in the second part in order to guarantee 2-connectivity of each subnetwork.

We first find the maximum number of link-disjoint paths for each node pair such that all paths have lengths not exceeding $R_{\max }$. We compute these paths in two stages. First, the unconstrained max-flow problem is solved, i.e., for each node pair the maximum number of flows that can be carried between these nodes is found such that the total length of all used links is minimized. At the second stage, link-disjoint paths passing through the links with positive flows in the first stage are found subject to the constraint that all paths have lengths not exceeding $R_{\max }$. These steps are repeated subsequently for all node pairs.

Suppose the network topology is represented by an undirected graph $G=(V, E)$, where $V$ is the node set and $E$ is the set of links. Let $P^{m n}$ denote the set of constrained link-disjoint paths found at the end of the first stage. Let $P_{k}^{m n}$ denote $k$-th shortest path in $P^{m n}$, and let $\left|P_{k}^{m n}\right|$ denote the number of links on path $P_{k}^{m n}$. Let the indicator function $\delta_{l n}$ describe the node-link adjacency defined as $\delta_{l n}=1$ if link $l$ is adjacent to node $n$.

We define the following binary decision variables: $Y_{n s}=1$ if node $n$ is in subnetwork $s$, and $U_{l s}=1$ if link $l$ is in subnetwork $s$. Next, we define the following auxiliary variables: $Z_{m n s}=1$ if $Y_{m s}=Y_{n s}=1$, i.e., both nodes $m$ and $n$ are in subnetwork $s, W_{k s}^{m n}=1$ if $P_{k}^{m n}$ lies completely inside subnetwork $s$, and $T_{s}=1$ if subnetwork $s$ exists.

The ILP formulation for the network partitioning problem is given by: Objective: Minimize $\sum_{s} T_{s}+\epsilon \sum_{s} \sum_{l} U_{l s}$ Subject to:

$$
\begin{gathered}
\sum_{s} U_{l s} \geq 1 \quad \forall l \\
Y_{n s} \geq U_{l s} \delta_{l n} \quad \forall n, \forall s, \forall l \\
Y_{n s} \leq \sum_{l \in E} U_{l s} \delta_{l n} \quad \forall n, \forall s \\
\sum_{l} U_{l s} \leq L_{m a x} \quad \forall s \\
\sum_{n}^{l} Y_{n s} \leq N_{m a x} \quad \forall s \\
Z_{m n s} \leq Y_{m s} \quad \forall(m, n), \forall s \\
Z_{m n s} \leq Y_{n s} \quad \forall(m, n), \forall s \\
Z_{m n s} \geq Y_{n s}+Y_{m s}-1 \quad \forall(m, n), \forall s \\
W_{k s}^{m n} \leq U_{l s} \forall l \in P_{k}^{m n}, \forall s, \forall k, \forall(m, n) \\
W_{k s}^{m n} \geq \sum_{l \in P_{k}^{m n}} U_{l s}-\left|P_{k}^{m n}\right|+1 \quad \forall s, \forall k, \forall(m, n) \\
\sum_{k} W_{k s}^{m n} \geq 2 Z_{m n s} \quad \forall(m, n), \forall s \\
U_{l s} \leq T_{s} \quad \forall l \\
Y_{n s}, U_{l s}, Z_{m n s}, W_{k s}^{m n} \in\{0,1\}
\end{gathered}
$$

The objective of the formulation is to minimize not only the total number of subnetworks, but also the total number of shared links. Both objectives are embedded into one objective function by multiplying the latter one with a small constant $\epsilon$ such that the minimization of the number of subnetworks has the high priority. For example, $\epsilon$ can be chosen as $\epsilon=1 /|V|$, where $|V|$ is the number of nodes. By reducing the number of shared links, we make the design of individual subnetworks more independent from each other.

Constraint (1) expresses that each link must belong to at least one subnetwork. Constraints (2-3) state that if one adjacent link of node $n$ belongs to subnetwork $s$, then node $n$ is also part of subnetwork $s$. Constraints (4-5) guarantee that each subnetwork satisfies node and link size constraints. Equations (6-8) imply that if both nodes $m$ and $n$ are in subnetwork $s$, then the variable $Z_{m n s}=1$, otherwise $Z_{m n s}=0$. Constraints (9-10) indicate that in order to have $W_{k s}^{m n}=1$, all the links on path $P_{k}^{m n}$ should reside in subnetwork $s$, otherwise $W_{k s}^{m n}=0$. Equation (11) provides the 2-connectivity requirement for each subnetwork. If nodes $m$ and $n$ are in subnetwork $s$, i.e. $Z_{m n s}=1$, then there should be at least 2 link-disjoint paths between nodes $m$ and $n$ lying completely inside subnetwork $s$. If there are no such paths, $Z_{m n s}=0$. Finally, (12) ensures the existence of a subnetwork if at least one link belongs to that subnetwork. 


\subsection{Greedy Heuristic Partitioning Algorithm}

Due to the high computational complexity of solving the ILP problem, it may not be possible to obtain the solution for large networks within a run-time limit. Therefore, we propose a heuristic algorithm for planar network topologies. A planar graph can be drawn in the plane such that its edges intersect only at their common end-vertices. ${ }^{17}$ For a planar graph, the regions bounded by the edges are called faces. The links surrounding a face constitute a simple cycle which is 2-connected. Two faces are called adjacent if they share at least one edge. The number of faces of a connected planar graph $G=(V, E)$ is given by the Euler formula

$$
|F|=|E|-|V|+2
$$

where $|E|$ is the number of edges and $|V|$ is the number of nodes in $G$.

The heuristic algorithm can be described in two parts. In the first part, we find all $|F|$ faces of the planar graph corresponding to the network topology. There are several algorithms for finding the faces in a planar graph. ${ }^{18}$ After faces are found, we combine adjacent faces to form the subnetworks which satisfy size constraints. The 2-connectivity constraint is met since individual faces and combinations of adjacent faces are 2-connected.

\subsubsection{Combining Faces}

Before stating the algorithm used in the heuristic algorithm, we make the following definition.

Definition: For a given graph $G$, let $P_{1}, P_{2}, \ldots, P_{K}$ be the set of all link-disjoint paths between nodes $m$ and $n$ that are computed as described in Section 3. Let $\left|P_{i}\right|$ denote the length of $P_{i}$. The dilation of $m$ and $n$ in topology $G$ is defined as

$$
\operatorname{dil}(m, n \mid G)=\frac{\max _{i=1,2, \ldots, K}\left|P_{i}\right|}{\min _{i=1,2, \ldots, K}\left|P_{i}\right|} .
$$

The face combining algorithm starts with a cluster which is initially a single face, and then begins to enlarge the cluster by combining it with adjacent faces. At each step, a candidate cluster is formed by joining the current cluster with one of its adjacent faces such that the newly formed cluster satisfies all size constraints. For all node pairs in each candidate cluster, all link-disjoint paths are calculated, and the average dilation is computed for each candidate cluster where the average is taken over all node pairs in the cluster. Among all candidate clusters, the one with the minimum average dilation is chosen, and the corresponding adjacent face is added to the current cluster to form the new cluster.

We continue enlarging the current cluster until no candidate cluster can be found. Then a new cluster is initiated with a face which is not included in any other cluster, and the same process is repeated until all faces are included in some cluster. The clusters obtained at the termination of the algorithm form the subnetworks.

The algorithm is described as follows:

1. Initiate a new cluster with an arbitrarily selected face which is not included in any other cluster. If no such face exists, stop.

2. For the current cluster find the candidate cluster that has the minimum average dilation. If no candidate cluster exists, go to Step 1.

3. Set current cluster to the candidate cluster found in Step 2, and go to Step 2.

\subsection{Numerical Results}

ILP formulation and the greedy heuristic algorithm are applied to two different network topologies. The 32-node topology given in Figure 5 is an approximation of a carrier's core network where nodes correspond to major US cities. ${ }^{13}$ The 23-node mesh network shown in Figure 1 is the right-half of the 32-node topology. The lengths of the links in these networks are the actual distances between major cities corresponding to nodes.

For the 23-node network various kinds of size constraints were used in the partitioning process. We have used the same constraints for both the ILP formulation and the heuristic algorithm. The ILP formulation was implemented on CPLEX 7.1. For $R_{\max }=2500 \mathrm{~km}, N_{\max }=12$ and $L_{\max }=15$, partitionings with both methods 
for the 23-node network are shown in Figures 1 and 3(a). The ILP formulation generated 3 subnetworks whereas the heuristic algorithm produced 4 subnetworks. When we increase $R_{\max }$ from $2500 \mathrm{~km}$ to $3000 \mathrm{~km}$ and leave the other parameters same as the previous example, the ILP formulation produces again the same 3 subnetworks, while the heuristic algorithm generates 4 subnetworks as shown in Figure 3(b).

On the other hand, with the parameters $R_{\max }=2500 \mathrm{~km}, N_{\max }=16$, and $L_{\max }=24$, the partitionings produced by the two methods look similar as shown in Figures 2(a) and 4(a): both methods produce 3 subnetworks. When we increase $R_{\max }$ to $3000 \mathrm{~km}$, a slight difference in partitioning with the heuristic algorithm occurs as can be seen in Figure 4(b), where the number of the subnetworks does not change, however their sizes are altered. With the same parameters, the output of the ILP formulation changes significantly as shown in Figure 2(b) where two subnetworks are generated with sizes of 11 and 15 nodes leading to a significant reduction in the objective cost, i.e., both the number of subnetworks and the number of shared links are reduced.

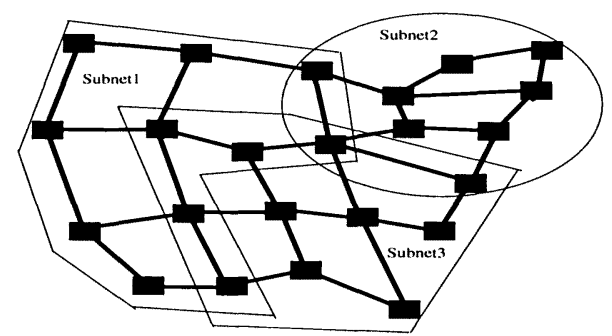

Figure 1. Network partitioning for 23-node network (ILP solution) with $R_{\max }=2500 \mathrm{~km}, L_{\max }=15, N_{\max }=12$, and with $R_{\max }=3000 \mathrm{~km}, L_{\max }=15, N_{\max }=12$

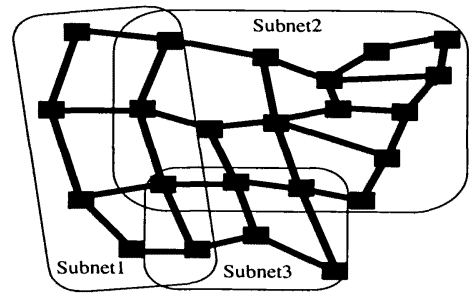

(a)

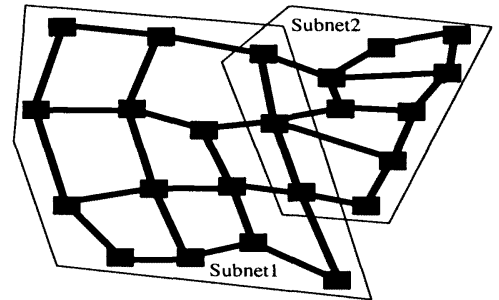

(b)

Figure 2. Network partitioning for 23-node network (ILP solution) with $L_{\max }=24, N_{\max }=16$ (a) $R_{\max }=2500 \mathrm{~km}$ (b) $R_{\max }=3000 \mathrm{~km}$

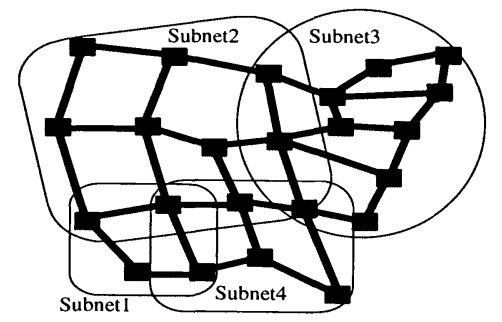

(a)

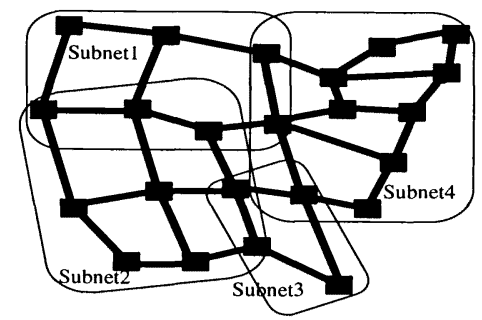

(b)

Figure 3. Network partitioning for 23-node network (Heuristic solution) with $L_{\max }=15, N_{\max }=12$ (a) $R_{\max }=2500 \mathrm{~km}$ (b) $R_{\max }=3000 \mathrm{~km}$

For the 32-node network, two sets of size constraints were used: $R_{\max }=3000 \mathrm{~km}, N_{\max }=16$, and $R_{\max }=$ $3000 \mathrm{~km}, L_{\max }=24$ and $N_{\max }=12$ and $L_{\max }=15$. The ILP formulation was not able to generate a reasonable 


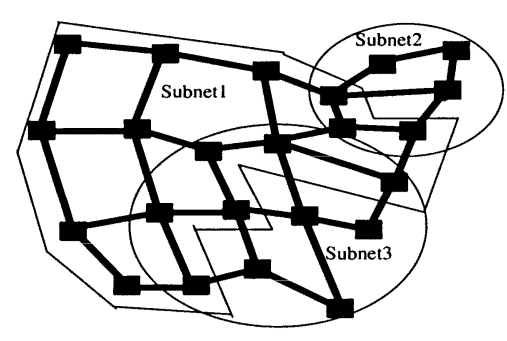

(a)

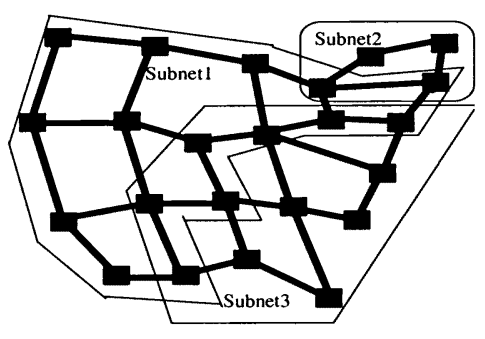

(b)

Figure 4. Network partitioning for 23-node network (Heuristic solution) with $L_{\max }=24, N_{\max }=16$ (a) $R_{\max }=2500 \mathrm{~km}$ (b) $R_{\text {max }}=3000 \mathrm{~km}$

solution within a runtime of 36 hours. The greedy heuristic algorithm produced a partitioning with 5 subnetworks shown in Figure 5 (a) for $N_{\max }=16$ and $L_{\max }=24$ case. With the parameters $N_{\max }=12$ and $L_{\max }=15$, the algorithm generated 6 subnetworks as shown in Figure 5 (b). We observe from the solutions of the heuristic algorithm that it is inclined to build a very large subnetwork close to size constraints. This can be explained by the greedy nature of the heuristic, i.e., it tries to enlarge current subnetwork as much as possible without considering the rest of the network topology.

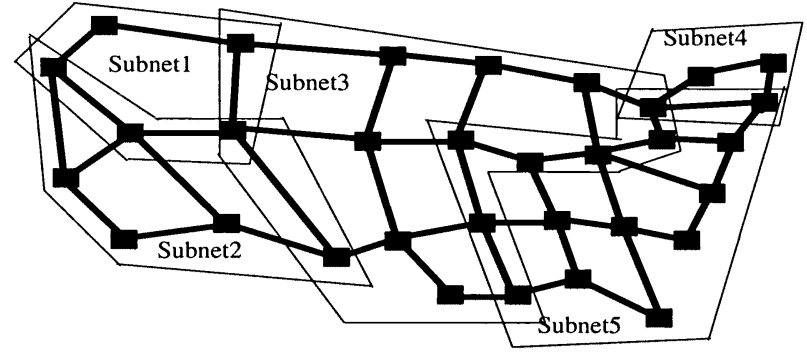

(a)

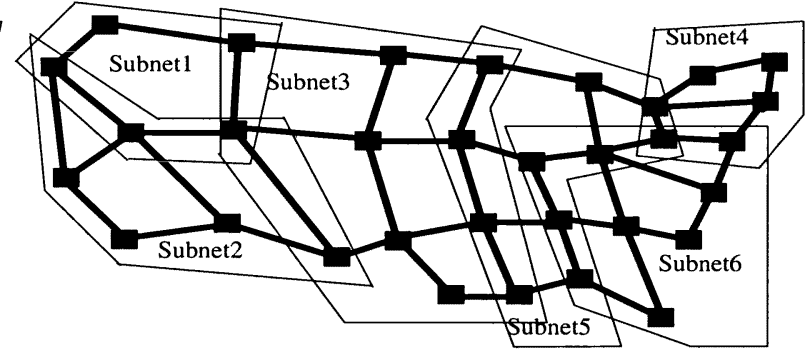

(b)

Figure 5. Network partitioning for 32-node network (Heuristic solution) with (a) $L_{\max }=24, N_{\max }=16 R_{\max }=3000 \mathrm{~km}$, (b) $L_{\max }=15, N_{\max }=12 R_{\max }=3000 \mathrm{~km}$

\section{DESIGN OF WORKING AND RESTORATION CAPACITIES}

In the previous section, we have partitioned the network for the overlapped subnetworks layer where some links may be shared between subnetworks. In this section, we first determine how these shared links are partitioned in the restoration layer, i.e., for each shared link the RM in charge of the recovery of paths passing through that link is determined. We call this problem the "Link Partitioning Problem". We will then analyze the capacity planning problem for the section restoration in translucent networks and present numerical results comparing fiber costs obtained with section restoration and path restoration.

\subsection{Link Partitioning in Restoration Layer}

A heuristic algorithm for the link partitioning problem is presented. Let $\Sigma_{l}$ denote the set of subnetworks sharing link $l$. For each subnetwork $S \in \Sigma_{l}$, all link-disjoint paths between all node pairs in $S$ are computed. Let $\tilde{P}_{l}^{S}$ denote the set of node pairs $(m, n), m, n \in S$ such that each such node pair has one of its link-disjoint paths passing through $l$. For each $(m, n) \in \tilde{P}_{l}^{S}$, we compute the average alternate path length, $\pi_{l}^{S}(m, n)$, over all link-disjoint paths except the one passing through $l$. We then compute the average alternate path length for subnetwork $S, \Pi_{l}^{S}$, which is given by

$$
\Pi_{l}^{S}=\frac{1}{\left|\tilde{P}_{l}^{S}\right|} \sum_{(m, n) \in \tilde{P}_{l}^{S}} \pi_{l}^{S}(m, n)
$$


where $\left|\tilde{P}_{l}^{S}\right|$ is the cardinality of set $\tilde{P}_{l}^{S}$. Link $l$ is then assigned in the restoration layer to subnetwork $S^{*}$ such that

$$
S^{*}=\arg \min _{S \in \Sigma_{l}} \Pi_{l}^{S} .
$$

This algorithm chooses the subnetwork which has the minimum average alternate path length. The idea behind this heuristic is that, alternate paths are restoration path candidates in case of failure of link $l$. The algorithm assigns $l$ to the subnetwork which has the minimum average length of restoration path candidates.

After forming the restoration layer, we proceed to the network design phase where working and restoration paths and wavelengths are selected, and appropriate link capacities are determined.

\subsection{Routing and Capacity Planning with Section Restoration}

We discuss the network design problem of determining working and restoration capacities so that the network traffic is fully restorable against all single-link failures. We assume that the network is partitioned into alloptical WSXC-based subnetworks. Although wavelength conversion is not possible inside subnetworks, it can be performed at the boundaries of the subnetworks. We further assume that the traffic demand is static, and there is one unit wavelength of traffic for each demand. The case where there are multiple units of traffic between some node pairs can also be modelled in this framework by placing multiple one-unit traffic between those nodes. We also assume that the working and restoration fibers are separate. We consider two different design approaches:

- Joint Design: All link capacities are optimized for the entire network, i.e., all subnetworks are jointly designed for both working and restoration capacities.

- Separate Design: First, working paths and capacities are designed for the entire network independent of partitioning of the network and restoration capacities. Then, for each subnetwork restoration capacities are computed considering all single-link failures. This design is carried out for each subnetwork independent of other subnetworks. The restoration capacity for link $l$ is determined by taking the maximum capacity assignment made on link $l$ among all subnetworks sharing $l$.

The optimum solution of the joint design has lower cost than the separate design. However, the computational complexity of obtaining the solution for joint design is much larger than the separate design. As the number of nodes in the network increases, solving the joint design optimally becomes extremely difficult since computational complexity is growing exponentially. As the number of nodes increases, number of subnetworks also increases, but subnetwork sizes remain limited since they are determined by the size constraints. Hence the separate design approach is more scalable.

\subsubsection{Joint Design}

An ILP formulation is presented for planning the network by jointly considering the working and restoration capacities. The formulation presented here is a path based formulation, so the working and restoration paths are predetermined. We first find the set of $K$-shortest paths, not necessarily link-disjoint, for all demand pairs. These paths are the candidates for working paths. Each path is then divided into sections such that all links on each section are assigned to the same subnetwork in the restoration layer. We perform this operation in such a way that all sections are maximal, i.e., no two sections can be combined. For the failure of each link, restoration path candidates for each failed connection are computed between the entry and exit nodes of the subnetwork which contains the failed link. Inside this subnetwork, all candidate restoration paths are link-disjoint from the failed path. However, restoration paths have exactly the same sections as the failed path except for the failed section.

Let $P^{s d}$ denote the set of $K$-shortest paths for demand pair $(s, d)$, and let $P_{k}^{s d}$ be the $k$-th shortest path in $P^{s d}$. Each path is divided into its sections given by

$$
P_{k}^{s d}=\bigcup_{i=1}^{t_{k}^{s d}} P_{k i}^{s d}
$$


where $P_{k i}^{s d}$ is the $i$-th section of $P_{k}^{s d}$, and $t_{k}^{s d}$ is the number of sections on $P_{k}^{s d}$. For each $l \in P_{k}^{s d}, R_{l k j}^{s d}$ denotes the $j$-th restoration path for $P_{k}^{s d}$ in case of failure of link 1 . Let $d_{l}$ denote the length of link $l$, and the number of wavelengths per fiber is denoted by $W$.

We define the following indicator functions: $\beta_{l k}^{s d}=1$ if $P_{k}^{s d}$ passes through link $l, \gamma_{\bar{l} k j l}^{s d}=1$ if $R_{\bar{l} k j}^{s d}$ passes through link $l$, and $\xi_{l k i}^{s d}=1$ if $P_{k i}^{s d}$ passes through link $l$. Binary decision variables are given by $Z_{k}^{s d}=1$ if $P_{k}^{s d}$ is chosen as the working path for demand pair $(s, d), X_{k i w}^{s d}=1$ if wavelength $w$ is assigned for working path on $P_{k i}^{s d}$, and $Y_{l k j w}^{s d}=1$ if $R_{l k j}^{s d}$ is assigned as the restoration path using wavelength $w$ when link $l$ fails. Decision variables $W C_{l}$ and $R C_{l}$ correspond to the number of fibers on link $l$ used by working and restoration paths, respectively.

The optimum joint network design problem is given by

Objective: Minimize $\sum_{l}\left(W C_{l}+R C_{l}\right) d_{l}$

Subject to:

$$
\begin{gathered}
\sum_{k} Z_{k}^{s d}=1 \quad \forall(s, d) \\
\sum_{w} X_{k i w}^{s d}=Z_{k}^{s d} \quad \forall(s, d), \forall k, \forall 1 \leq i \leq t_{k}^{s d} \\
\sum_{j} \sum_{w=1}^{W} Y_{l k j w}^{s d}=Z_{k}^{s d} \quad \forall(s, d), \forall k, \forall l \in P_{k}^{s d} \\
\sum_{(s, d)} \sum_{k} \sum_{i=1}^{t_{k}^{s d}} X_{k i w}^{s d} \xi_{l k i}^{s d} \leq W C_{l} \quad \forall l, \forall w \\
\sum_{(s, d)} \sum_{k} \sum_{j} \beta_{\bar{l} k}^{s d} \gamma_{\bar{l} k j l}^{s d} Y_{\overline{l k j w}}^{s d} \leq R C_{l} \quad \forall l, \forall w, \forall \bar{l} \neq l \\
Z_{k}^{s d}, X_{k i w}^{s d}, Y_{l k j w}^{s d} \in\{0,1\} \\
W C_{l}, R C_{l} \in Z^{+}
\end{gathered}
$$

The objective of this formulation is to minimize the cost of total fiber used. Constraint (13) ensures that among all working path candidates exactly one is chosen. In (14), it is stated that for every section of a working path a wavelength should be assigned. This constraint allows that wavelength conversion can be performed at the endpoints of each section. Constraint (15) implies that for every link failure on a working path there is a restoration path assigned. Constraints (13-15) are the path and wavelength assignments for the formulation. Constraints (16-17) are link capacity assignments, i.e., links are assigned sufficient working and restoration capacities for carrying all traffic demands and for achieving full restorability against all single-link failures.

\subsubsection{Separate Design}

The formulation for separate design is the decoupled version of the joint formulation. Working and restoration path/capacity assignment problems are separated and solved sequentially. Furthermore, the restoration part of the problem is partitioned into multiple subproblems, i.e., design of restoration capacities for each subnetwork is carried out separately. The separate design formulation is not presented here due to space limitations.

\subsection{Numerical Results}

Joint and separate design methods are applied to 23 and 32-node mesh networks. For each network topology, 4 different sets of traffic demands are generated. We use the following parameters: number of candidate paths for working paths $K=4$, and number of wavelengths $W=8$. The ILP formulation was implemented on CPLEX 7.1 .

Both partitionings obtained by the ILP formulation and heuristic algorithm for the 23-node network were considered. For the joint design algorithm, the maximum running time of CPLEX was limited to 36 hours. For the 23-node network, joint design produced suboptimum solutions within the run-time limit. For the 32-node network, joint design was not able to generate a reasonable integer feasible solution within the run-time limit for all demand sets. With the separate design, working part of the problem generated suboptimal solutions within 36 hours of running time, whereas the optimum solution was obtained in the restoration part. 
In order to evaluate the performance of section restoration, we compare it with path rerouting. With path rerouting in all-optical networks, we assume that working and restoration paths are mutually link-disjoint. For the path restoration, there are two cases to be considered with respect to the locations of the transponders. First, in order to have a fair comparison with section restoration, we locate the transponders at the same locations as in the section restoration case. In this case, wavelength conversion can be exercised only at the boundaries of subnetworks. For the second case, in order to compute a lower bound on the network cost, we discuss path restoration with wavelength conversion at all nodes of the network. We call the former case as fair conversion and latter case as the full conversion. In path restoration with fair and full conversion, network design problems are formulated as ILP problems, and joint and separate design methods are used for designing subnetworks as in the case of section restoration. Because of space limitations, these formulations are not presented here.

For the 23-node network four demand sets have 63,67, 69, and 63 demand pairs, respectively, are randomly generated. With optimum ILP partitioning and parameters $R_{\max }=2500 \mathrm{~km}, L_{\max }=24$ and $N_{\max }=16$, the fiber costs for section restoration and path restoration/fair conversion and path restoration/full conversion cases are shown for joint and separate designs in Table 1.

Table 1. Total fiber costs for the 23-node network partitioned by ILP formulation with $R_{\max }=2500 \mathrm{~km} N_{\max }=16$ and $L_{\max }=24$ (*: suboptimal).

\begin{tabular}{||c||c|c||c|c||c|c||}
\hline \hline \multirow{2}{*}{$\begin{array}{c}\text { Demand } \\
\text { set }\end{array}$} & \multicolumn{2}{c||}{ Section } & \multicolumn{2}{c||}{ Path (Fair) } & \multicolumn{2}{c||}{ Path (Full) } \\
\cline { 2 - 7 } & Joint & Separate & Joint & Separate & Joint & Separate \\
\hline \hline 1 & $24136^{*}$ & 22124 & 22275 & 19761 & 19327 & 19641 \\
\hline 2 & 24838 & 25347 & $26396^{*}$ & 22478 & $22821^{*}$ & 22478 \\
\hline 3 & 22715 & 24280 & $23934^{*}$ & 22057 & 21361 & 21784 \\
\hline 4 & 21581 & 23866 & 21095 & 21580 & 20391 & 20771 \\
\hline \hline
\end{tabular}

We observe from Table 1 that with all three restoration techniques there are cases where separate design generates lower fiber cost than joint design. This is due to the fact that the results marked with "*" are suboptimal that are obtained within the 36-hour runtime limit. Decoupled subnetwork design problems in separate design have lower computational complexity, and they may generate better results within the time limits. In general, for all restoration methods costs for the joint and separate designs are close. An important observation is that the largest difference between fiber costs obtained with section restoration/separate design and path restoration/fair conversion is $13.1 \%$. The above difference increases to $17.0 \%$ if we allow full conversion in path restoration.

The above analysis is repeated for the 23-node network partitioned with the heuristic algorithm, and the results are shown in Table 2. These results present similar characteristics with those for the ILP partitioned network. Since in path restoration/full conversion case network cost is independent of partitioning, same fiber costs are obtained in that case as those in Table 1. With this partitioning, the largest difference between fiber costs obtained with section restoration/separate design and path restoration/fair conversion is $3.0 \%$. The above difference increases to $9.5 \%$ if we allow full conversion in path restoration. Both numbers are smaller than those obtained with the ILP partitioned network.

We repeat the above experiments for the 32-node network which is partitioned by the heuristic algorithm with parameters $R_{\max }=3000 \mathrm{~km}, L_{\max }=24$, and $N_{\max }=16$. Four demand sets with $89,73,86$, and 66 demand pairs, respectively, are randomly generated. Fiber costs for section restoration/separate design, path restoration/fair conversion/separate design, and path restoration/full conversion for joint and separate designs are given in Table 3 .

Fiber costs of section restoration/separate design, and path restoration/fair conversion/separate design are close to each other, and the highest difference is $7.3 \%$. This difference remains unchanged when full wavelength conversion is allowed for path restoration. 
Table 2. Total fiber costs for the 23-node network partitioned by the heuristic algorithm with $R_{\max }=2500 \mathrm{~km} N_{\max }=16$ and $L_{\max }=24(*$ : suboptimal).

\begin{tabular}{||c||c|c||c|c||c|c||}
\hline \hline \multirow{2}{*}{$\begin{array}{c}\text { Demand } \\
\text { set }\end{array}$} & \multicolumn{2}{c||}{ Section } & \multicolumn{2}{c||}{ Path (Fair) } & \multicolumn{2}{c||}{ Path (Full) } \\
\cline { 2 - 7 } & Joint & Separate & Joint & Separate & Joint & Separate \\
\hline \hline 1 & $20944^{*}$ & 20340 & $19865^{*}$ & 19764 & 19327 & 19641 \\
\hline 2 & $24183^{*}$ & 24125 & $25979^{*}$ & 23821 & $22821^{*}$ & 22478 \\
\hline 3 & $23174^{*}$ & 23381 & $23858^{*}$ & $23585^{*}$ & 21361 & 21784 \\
\hline 4 & $21453^{*}$ & 20440 & $21432^{*}$ & $20825^{*}$ & 20391 & 20771 \\
\hline \hline
\end{tabular}

Table 3. Total fiber costs for the 32-node network partitioned by the heuristic algorithm with $R_{\max }=3000 \mathrm{~km} N_{\max }=16$ and $L_{\max }=24$ (*: suboptimal).

\begin{tabular}{||c||c||c||c|c||}
\hline \hline \multirow{2}{*}{$\begin{array}{c}\text { Demand } \\
\text { set }\end{array}$} & \multicolumn{1}{c||}{ Section } & Path (Fair) & \multicolumn{2}{c||}{ Path (Full) } \\
\cline { 2 - 5 } & Separate & Separate & Joint & Separate \\
\hline \hline 1 & 45212 & 42129 & $44452^{*}$ & 42107 \\
\hline 2 & 40360 & 39303 & $39230^{*}$ & 36903 \\
\hline 3 & 44151 & 43856 & $41957^{*}$ & 41974 \\
\hline 4 & 36221 & $37416^{*}$ & $35724^{*}$ & 35256 \\
\hline \hline
\end{tabular}

The number of transponders used by translucent networks with section section restoration and opaque networks are shown in Table 4 for the 32-node network with the network partitioning shown in Figure 5. The results show that the transponder cost is substantially reduced for the translucent network compared to the opaque network.

Table 4. Total number of transponders for translucent and opaque networks with the 32-node network partitioned by the heuristic algorithm with $R_{\max }=3000 \mathrm{~km} N_{\max }=16$ and $L_{\max }=24$.

\begin{tabular}{||c|c|c||}
\hline \hline Demand set & Translucent & Opaque \\
\hline \hline 1 & 320 & 1830 \\
\hline 2 & 295 & 1670 \\
\hline 3 & 312 & 1828 \\
\hline 4 & 280 & 1632 \\
\hline \hline
\end{tabular}

\section{CONCLUSIONS}

The network partitioning problem in translucent optical networks is studied as an optimization problem, and an ILP formulation for the problem is presented. Due to the high computational complexity of the ILP optimization, we present an alternative greedy heuristic algorithm for planar networks that performs comparable with the ILP formulation in terms of the number of subnetworks in the partitioning.

Numerical results demonstrate that the section restoration with separate design produces fiber costs that are generally within $10 \%$ of those with path restoration/joint design when fair conversion is used. Moreover, fiber costs with section restoration are also within $15 \%$ of those with path restoration/full conversion. It is also observed that fiber costs with section restoration with separate design are $4-16 \%$ lower when the network partitioned with the heuristic algorithm is used. 
It is also shown that the transponder cost is substantially reduced in translucent optical networks compared to opaque networks. Coupled with the relatively small increase in fiber costs, this huge reduction in transponder costs make translucent optical networks a viable architecture for future optical networks.

\section{ACKNOWLEDGMENTS}

This work was partially supported by the Scientific and Technological Research Council of Turkey (TUBITAK) under the project 199E005. The authors also would like to thank anonymous reviewers for many helpful comments.

\section{REFERENCES}

1. S. B. Alexander et al., "A Precompetitive Consortium on Wide-Band All-Optical Networks," J. Lightwave Technol., vol. 11, pp. 714-735, May 1993.

2. R. E. Wagner et al., "MONET: Multiwavelength Optical Networking," J. Lightwave Technol., vol. 14, pp. 1349-1355, June 1996.

3. J. M. H. Elmirghani and H. T. Mouftah, "All-Optical Wavelength Conversion: Technologies and Applications in DWDM Networks," IEEE Communications Magazine, vol. 38, pp. 86-92, March 2000.

4. E. Karasan and E. Ayanoglu, "Performance of WDM Transport Networks," IEEE Journal On Selected Areas In Communications, vol. 16, No. 7, pp. 1081-1096, September 1998.

5. J. M. Yates, M. P. Rumsewicz and J. P. R. Lacey, "Wavelength Converters in Dynamically-Reconfigurable WDM Networks," IEEE Commun. Surveys, 2 Q1999.

6. B. Ramamurthy and B. Mukherjee, "Wavelength Conversion in WDM Networking," IEEE Journal On Selected Areas In Communications, vol. 16, No. 7, pp. 1061-1073, September 1998.

7. K. Bala, R. Cordell and E. Goldstein, "The Case for Opaque Multiwavelength Lightwave networks," Proc. IEEE/LEOS Summer Topical Meeting on Global Info. Infrastructure, Keystone, CO, Aug.1995.

8. P. Green, "Progress in Optical Networking," IEEE Communications Magazine, vol. 39, pp. 54-61, January 2001.

9. B. Rajagopalan et al., "IP over Optical Networks: Architectural Aspects," IEEE Communications Magazine, vol. 38, pp. 94-102, September 2000.

10. J. Strand, A. L. Chiu and R. Tkach, "Issues for Routing in the Optical Layer," IEEE Communications Magazine, vol. 39, pp. 81-87, February 2001.

11. H. Zang, "Sub-Path Protection for Scalability and Fast Recovery in WDM Mesh Networks," OFC'2002, ThO6, Feb. 2002.

12. P-H. Ho and H. Mouftah, "A Framework for Service-Guaranteed Shared Protection in WDM Mesh Networks," IEEE Communications Magazine, vol. 40, no. 2, pp. 97-103, Feb. 2002.

13. M. Alanyali and E. Ayanoglu, "Provisioning Algorithms for WDM Optical Networks," IEEE/ACM Transactions on Networking, vol. 7, no. 5, pp.767-778, Oct. 1999.

14. G. Mohan and C. S. R. Murthy, "Lightpath Restoration in WDM Optical Networks," IEEE Network, vol. 14, pp. 24-32, November/December 2000.

15. R.R. Iraschko and W.D. Grover, "A Highly Efficient Path-restoration Protocol for Management of Optical Network Transport Integrity," IEEE Journal On Selected Areas In Communications, vol. 18, No. 5, pp. 779794, May 2000.

16. B. T. Doshi, S. Dravida, P. Harshavardhana, O. Hauser and Y. Wang, "Optical Network Design and Restoration," Bell Labs Technical Journal, pp. 58-84, January-March 1999.

17. Béla Bollobás, Graph Theory. Springer-Verlag, NY, 1979.

18. T. Nishizeki and N. Chiba, Planar Graphs: Theory and Algorithms. North Holland, 1988. 